\title{
Device based prevention of neurological events in coronary artery bypass patients with calcified ascending aorta.
}

\author{
Michal Szlapka ${ }^{1}$, Philipp Peitsmeyer ${ }^{1}$, Stefanie Halder ${ }^{1}$, Oliver Natho ${ }^{1}$, Michael Lass ${ }^{1}$, and \\ Thorsten Hanke ${ }^{1}$ \\ ${ }^{1}$ Asklepios Klinikum Harburg
}

September 24, 2021

\begin{abstract}
Patients with severely calcified aorta undergoing conventional cardiac surgery are at increased risk for postoperative neurologic deficits. Implementation of cerebroprotective devices may substantially reduce or even eliminate the risk of adverse neurologic event, thus enabling surgical therapy, especially when interventional treatment cannot be considered an alternative option.
\end{abstract}

\section{Introduction}

In patients undergoing coronary artery bypass grafting, presence of severe aortic calcifications may substantially increase risk of perioperative stroke or even preclude surgery. Therefore, strategies aiming at minimizing risk of perioperative neurologic deficit must be applied. Among measures against perioperative stroke, implementation of intravascular devices capturing aortic debris appears safe and simple methods. Based on presented case, the benefits of intravascular neuroprotective device have been shown.

Institutional Review Board (IRB) approval was not applicable in refer to presented manuscript.

\section{Case report}

A 72-year-old patient, suffering from exertional angina symptoms, was admitted to our clinic to undergo elective cardiological evaluation. Upon coronary angiography, a triple vessel disease with a significant left main stenosis and chronic total occlusion (CTO) of the right coronary artery (RCA) was confirmed, summing up to a Syntax Score of 31 points. Hence, a high lesion complexity was confirmed and multidisciplinary evaluation of existing therapy options was in favor of surgery over percutaneous intervention. (Video 1). Therefore, the patient was scheduled for elective coronary artery bypass grafting (CABG). In course of further preoperative work-up, echocardiography revealed normal left ventricular function and excluded valvular pathologies. However, a substantial dilation of aortic root was suspected, prompting further evaluation with use of computed tomography (CT). Though CT scans showed normal aortic dimensions, it revealed severe calcifications of the ascending aorta (Figure 1). Possible treatment options were thoroughly discussed by institutional heart team, which recommended CABG under implementation of neuroprotective device Claret Sentinel (Boston Scientific, USA). Thorough analysis of CT scan images allowed for identification of aortic areas with less calcification burden, amenable for installation of cardiopulmonary bypass and cross-clamp (Figure 2).

Prior to chest opening and after heparinization with an activated clotting time of more than 250 seconds, a cerebroprotective device Claret Sentinel device was placed in the innominate and left carotid artery through fluoroscopy guided puncture of the right radial artery (Video 2).

Following placement of the Claret device, the chest was opened through a median sternotomy. Based on preoperative computed tomography and intraoperative inspection, aortic areas with less calcification burden 
were chosen for cardiopulmonary bypass canulation and application of aortic cross-clamp.

Cerebral activity was monitored throughout the surgery with use of near infrared spectroscopy (NIRS). Proper and unaffected cerebral perfusion was demonstrated at all times, especially during aortic cannulation, as well as implementation and release of aortic cross clamp.

During procedure, arterial revascularization of left anterior descending (LAD) and circumflex (CX) territories was performed with use of the left mammary artery and left radial artery, respectively. Sufficiently collateralized, but severely calcified posterolateral branch of the totally occluded right coronary artery was considered unsuitable for grafting.

Removal of the Claret device preceded weaning off cardiopulmonary bypass. Certain amount of fine-grained debris was found in both device baskets (Figure 3).

Patient experienced an uneventful postoperative course and was discharged on the $7^{\text {th }}$ postoperative day. Neuro-MRI in a fluid attenuation inversion recovery (FLAIR) mode prior to discharge demonstrated intact cerebral function and excluded procedure-related ischemic lesions, thus confirming sufficient neuroprotection provided by the Claret device. The results of magnetic resonance imaging correlated well with postoperative neurological patient status. (Video 3).

Within first 30 days after surgery, no adverse cardiovascular or neurologic events occurred. Further follow-up visits in outpatient clinic have been scheduled.

\section{Discussion}

Currently, interventional therapies have been increasingly implemented for treatment of even complex cardiovascular disorders. Regarding coronary artery disease, percutaneous interventions have recently been used alternatively for treatment of certain coronary lesions, but complex stenoses with high Syntax Score remain a domain of surgery ${ }^{1}$.

Though calcification of the ascending aorta may often accompany cardiac diseases in adults, especially in advanced age, incidence of true porcelain or egg-shell aorta has usually been overestimated. It may result from lack of established diagnostic criteria, allowing for adequate evaluation of calcification severity and its impact on perioperative neurologic risk. Therefore, many patients may be denied cardiac surgery.

Recently, Snow and colleagues developed CT-scan-based classification of aortic calcification. Precise analysis of location and extent of calcifications allowed, among others, for estimation of real prevalence of 'true' porcelain aorta, being reported in approximately $0.6 \%$ patients undergoing cardiac surgery ${ }^{2}$. Snow's classification system distinguishes 6 severity grades, from $0=$ normal aorta to $5=$ porcelain aorta with complete circumferential calcifications. In presented patient, CT scan revealed advanced aortic involvement up to $74 \%$ of analyzed segments, corresponding with grade 3 of the above-mentioned classification.

Whenever severe aortic calcification in a patient supposed to undergo cardiac surgery is discovered, a question regarding risk of perioperative stroke and operability arises. In coronary artery surgery, several approaches allowing for avoidance of aortic manipulation have been recommended, including peripheral arterial canulation of femoral or axillary artery, canulation of innominate artery, CABG on fibrillating heart or off-pump procedures $^{3}$. However, each of them poses some technical challenges. In addition, off-pump coronary artery bypass grafting was shown to be related to suboptimal clinical results in a mid-term observation ${ }^{4}$.

A strategy used in presented patient combined surgical revascularization and percutaneous placement of neuroprotective device Claret Sentinel. Its implementation has been reported in large series of patients undergoing transcatheter aortic valve implantation (TAVI), though in TAVI population no clear clinical benefit has been demonstrated ${ }^{5}$. In a randomized study assessing effectiveness of neuroprotective devices in patients undergoing surgical aortic valve replacement (SAVR), no benefit in terms of reduction of postoperative stroke rate could be shown either ${ }^{6}$. However, certain potential for lowering the incidence of postoperative delirium and cognitive deficits has been confirmed. Hypothetically, there may exist a different mechanism leading to cerebral emboli in patients undergoing aortic valve replacement and those receiving bypasses at the presence 
of severely calcified aorta. Whereas dislodged debris from excised aortic valve may remain hidden within left ventricle trabeculations and be ejected already after removal of Claret device, aortic calcifications in CABG patients seem to be 'activated' at the very moment of aortic manipulation (canulation and cross clamp). Further imaging studies would be useful to validate this presumption.

In presented patient, use of Claret device has been shown to be a simple but effective measure against neurological complication. In addition, implantation of neuroprotective device allowed for avoidance of more complex, technically challenging methods of cerebral protection. Among existing neuroprotective strategies, implementation of Claret Sentinel device appears relatively simple method to capture microemboli and debris from a mechanically manipulated calcified aorta. Further studies with sufficient sample size would surely help examine the role and true clinical impact of Claret Sentinel device in protecting CABG patients from embolic neurological events.

\section{References}

1. Neumann FJ, Sousa-Uva M, Ahlsson A, et al. 2018 ESC/EACTS Guidelines on myocardial revascularization. Eur Heart J 2019;40(2):87-165.

2. Snow T, Semple T, Duncan A, et al. 'Porcelain aorta': a proposed definition and classification of ascending aortic calcification. Open Heart 2018;5(1):e000703.

3. Osaka S, Tanaka M. Strategy for Porcelain Ascending Aorta in Cardiac Surgery. Ann Thorac Cardiovasc Surg 2018;24(2):57-64.

4. Shroyer AL, Hattler B, Wagner TH, et al. Five-Year Outcomes after On-Pump and Off-Pump CoronaryArtery Bypass. N Engl J Med 2017;377(7):623-632.

5. Kapadia SR, Kodali S, Makkar R, et al. Protection Against Cerebral Embolism During Transcatheter Aortic Valve Replacement. J Am Coll Cardiol 2017;69(4):367-377.

6. Mack MJ, Acker MA, Gelijns AC, et al. Effect of Cerebral Embolic Protection Devices on CNS Infarction in Surgical Aortic Valve Replacement: A Randomized Clinical Trial. JAMA 2017;318(6):536-547.

\section{Figures}

Figure 1. Preoperative computed tomography, showing severe calcification of ascending aorta. Over $50 \%$ of analyzed aortic segments have been shown to be affected.

Figure 2. Aortic areas with less calcification burden have been identified as potentially suitable for cardiopulmonary bypass installation and aortic cross-clamp.

Figure 3. Following removal of Claret device, device baskets were inspected for debris. The amount of debris would have presumably been capable of causing immediate postoperative cerebral ischemic lesions. 

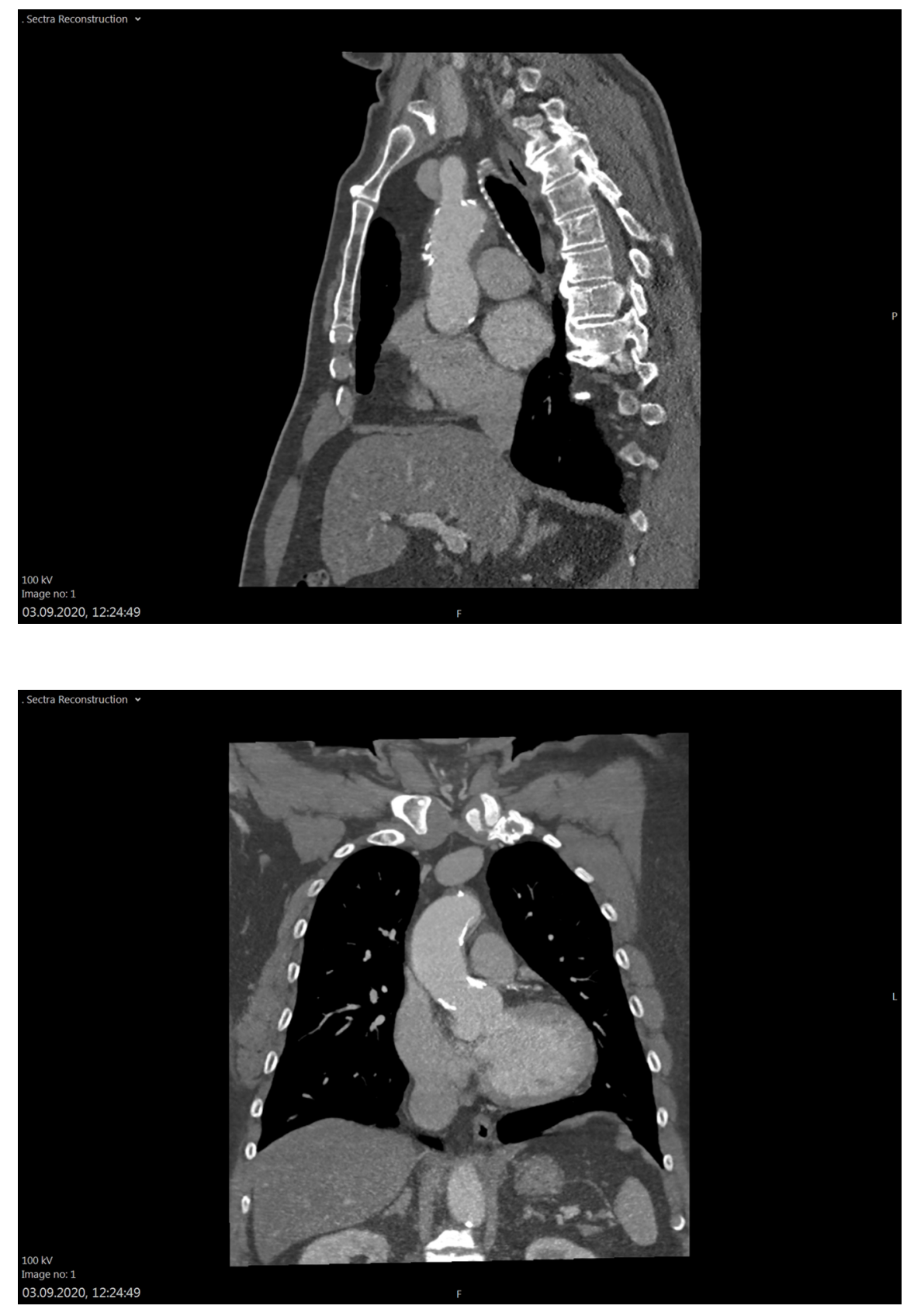


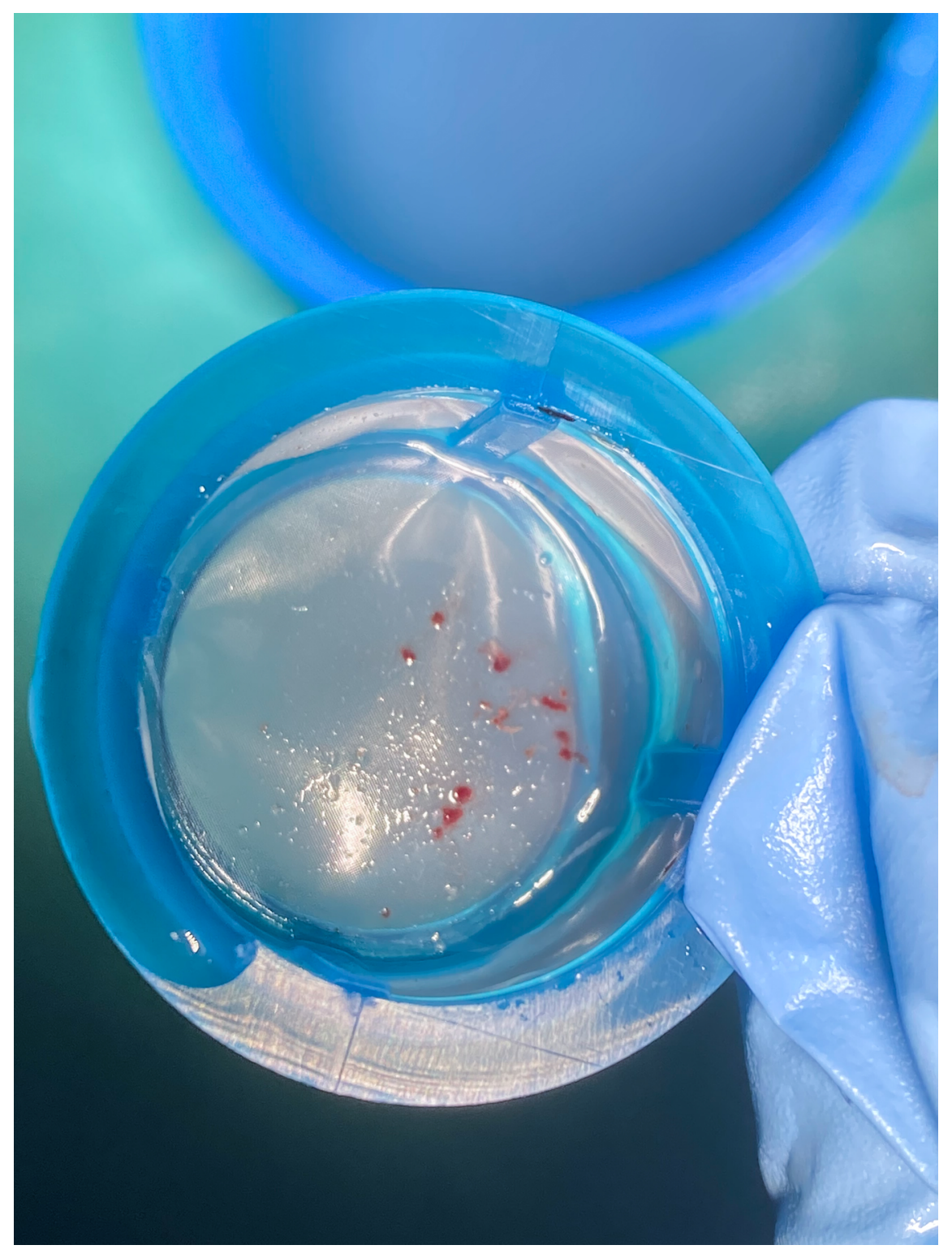

